\title{
Modal expansions and completeness relations for some time-dependent Schrödinger equations
}

\author{
Peter D. Miller*, N.N. Akhmediev ${ }^{1}$ \\ Australian Photonics Cooperative Research Centre, Optical Sciences Centre, \\ The Australian National University, Canberra, ACT 0200, Australia
}

\begin{abstract}
With the use of a variant of the method of separation of variables, the initial value problem for the time-dependent linear Schrödinger equation is solved exactly for a large class of potential functions related to multisoliton interactions in the vector nonlinear Schrödinger equation. Completeness of states is proved for absolutely continuous initial data in $L_{1}$. Copyright $($ ) 1998 Elsevier Science B.V.
\end{abstract}

PACS: 02.30.Jr; 03.40.Kf; 03.65.Ge

Keywords: Time-dependent Schrödinger equations; Exact solvability; Completeness relations

\section{Introduction}

The nonperturbative solution of the initial value problem for the linear Schrödinger equation

$$
\mathrm{i} \partial_{t} f+\frac{1}{2} \partial_{x}^{2} f-V(x, t) f=0
$$

subject to the initial condition $f(x, 0)=f_{0}(x)$ is a central problem of quantum mechanics in one space dimension, where $V(x, t)$ is the potential energy of a particle moving along the $x$-axis. In such quantum applications, the problem (1) for time-dependent potentials has not received nearly as much attention as the stationary case where $V=V(x)$. One reason for this is that if the potential is time-dependent at all, then often the physical mechanism responsible for the dynamics of the potential energy function couples $V$ to the wave function $f$ in a symmetrical way that leads to a nonlinear system. For example, the Maxwell-Bloch equations of quantum optics arise in this way. Exact solutions can be found for some of these nonlinear problems [1].

On the other hand, the linear equation (1) also comes up in the study of the classical optics of paraxial beams in planar waveguides. Here, $x$ and $t$ are both spatial coordinates in a planar dielectric medium; the function $f(x, t)$ is

\footnotetext{
* Corresponding author. Present address: School of Mathematics, Institute for Advanced Study, Princeton, NJ 08540, USA. Tel.: +1 (609) 734 8094; fax: +1 (609) 951 4459; e-mail: millerpd@math.ias.edu.

${ }^{1}$ Tel.: +61 (2) 6249 0191; fax: +61 (2) 6249 5184; e-mail: nna124@rsphysse.anu.edu.au.
} 
the slowly varying envelope of an electric field for a stationary monochromatic light beam propagating primarily in the $t$ direction, and $-V(x, t)$ is proportional to the spatial variation of the refractive index. In this case, unlike in onedimensional quantum mechanics, $t$ dependence in $V(x, t)$ without coupling to another system is natural because $V$ represents fixed inhomogeneities in a macroscopic medium. The time-dependent Schrödinger equation (1) is used frequently to model the behavior of passive linear integrated optical devices like waveguide tapers, waveguide junctions, and power splitters. Although $t$ dependence in the potential function $V$ is quite natural from the modern point of view of optical applications, it seems that many of the techniques currently used in optics are derived perturbatively from the more classical quantum mechanical theory of stationary potentials. These perturbative techniques are all variants of coupled mode theory. There would indeed be some value in developing alternative methods that could be used to study the time-dependent Schrödinger equation without introducing any more small parameters than are necessary to derive the equation to begin with.

A general strategy in treating the linear problem (1) nonperturbatively is to consider separating the time evolution from the spatial structure by seeking a family of particular solutions $\phi(x, t, \lambda)$ of (1) that for each $\lambda$ satisfy independent ordinary differential equations in $x$ and $t$. When they exist, these particular solutions are called the modes of the potential function $V(x, t)$. This procedure does not work for all potential functions $V(x, t)$. However, it is known to apply when the Schrödinger equation is stationary, that is, when the potential does not depend on $t$. Let us review the usual procedure of separation of variables, introducing a suggestive formalism. There are three steps:

1. Make an assumption about the form of the potential. Take $V(x, t)=g(x, t)+h(x, t)$.

2. Use this assumption to split the partial differential equation (PDE) into two ordinary differential equations (ODEs) with a parameter $\lambda^{2}$ playing the role of a separation constant. Thus, take

$$
\frac{1}{2} \partial_{x}^{2} \phi-g(x, t) \phi=-2 \lambda^{2} \phi, \quad \mathrm{i} \partial_{t} \phi-h(x, t) \phi=2 \lambda^{2} \phi .
$$

Introducing the vector $\mathbf{u}(x, t, \lambda)=\left(\phi, \partial_{x} \phi\right)^{\mathrm{T}}$, these ODEs can be written in the form

$$
\partial_{x} \mathbf{u}=\mathbf{L u}, \quad \partial_{t} \mathbf{u}=\mathbf{B u},
$$

where the matrices $\mathbf{L}$ and $\mathbf{B}$ are given by

$$
\mathbf{L}=\left[\begin{array}{cc}
0 & 1 \\
-4 \lambda^{2}+2 g(x, t) & 0
\end{array}\right], \quad \mathbf{B}=-\frac{1}{2}\left[\begin{array}{cc}
4 \mathrm{i} \lambda^{2}+2 \mathrm{i} h(x, t) & 0 \\
2 \mathrm{i} \partial_{x} h(x, t) & 4 \mathrm{i} \lambda^{2}+2 \mathrm{i} h(x, t)
\end{array}\right] .
$$

It is obvious that if for some $\lambda$ both ODEs can be simultaneously solved for a function $\phi(x, t, \lambda)$, then this function will also satisfy the PDE (1).

3. Demand that these two ODEs can be simultaneously solved for all $\lambda$. This will place constraints on the components $g(x, t)$ and $h(x, t)$ of the potential. The compatibility condition is $\partial_{x} \partial_{t} \mathbf{u}=\partial_{t} \partial_{x} \mathbf{u}$, or in terms of the matrices $\mathbf{L}$ and $\mathbf{B}$,

$$
\partial_{t} \mathbf{L}-\partial_{x} \mathbf{B}+[\mathbf{L}, \mathbf{B}]=\mathbf{0} .
$$

For the particular matrices above, this condition is equivalent to

$$
\partial_{t} g(x, t)=0, \quad \partial_{x} h(x, t)=0 .
$$

Thus, as is well-known, separation of variables in the usual sense works when the potential has the form $V(x, t)=$ $g(x)+h(t)$. Usually $h(t)$ is taken to be zero, and we then recover the family of stationary Schrödinger equations.

The main features of this familiar procedure are the following. First, the modes of the problem $\phi(x, t, \lambda)$ satisfy the PDE (1) but are obtained rather by finding simultaneous solutions to two compatible ODEs. Second, the modes 
are indexed by a complex parameter $\lambda$. The dependence on the parameter $\lambda$ means that there are often enough of these solutions to use superposition to construct the solution to the initial value problem for arbitrary initial data in some class. This completeness of states is one of the most important features of the separation of variables procedure.

Our aim is to present an alternative separation procedure within the same framework that applies to more interesting potential functions $V(x, t)$ and preserves the essential features of modes being obtained from ODEs and being complete. This means that the splitting of the Schrödinger equation must be such that the compatibility condition (5) admits a broader class of solutions. This can be accomplished by redesigning the separation scheme as follows:

1. Make a different assumption about the form of the potential. Choose any positive integer $N$. Introduce $N$ complex functions $q_{n}(x, t)$ making up a vector $\mathbf{q}(x, t)$, and suppose that the potential can be expressed as

$$
V(x, t)=-\mathbf{q}(x, t)^{\mathrm{T}} \mathbf{q}(x, t)^{*}=-\sum_{n=1}^{N}\left|q_{n}(x, t)\right|^{2} .
$$

2. Split the PDE into two ODEs, one in space and one in time, with a parameter $\lambda$. Let $\mathbf{u}(x, t, \lambda)$ be a vector of $N+1$ components, whose first component is $\phi(x, t, \lambda)$. Again consider the system (3) where this time the matrices $\mathbf{L}$ and $\mathbf{B}$ are given by

$$
\mathbf{L}=\left[\begin{array}{cc}
-2 \mathrm{i} \lambda & \mathbf{q}(x, t)^{\mathrm{T}} \\
-\mathbf{q}(x, t)^{*} & \mathbf{0}
\end{array}\right], \quad \mathbf{B}=-\frac{1}{2}\left[\begin{array}{cc}
4 \mathrm{i} \lambda^{2}+\mathrm{i} V(x, t) & -\left(2 \lambda+\mathrm{i} \partial_{x}\right) \mathbf{q}(x, t)^{\mathrm{T}} \\
\left(2 \lambda-\mathrm{i} \partial_{x}\right) \mathbf{q}(x, t)^{*} & \mathbf{i q}(x, t)^{*} \mathbf{q}(x, t)^{\mathrm{T}}
\end{array}\right] .
$$

It is not hard to see that if these two ODEs can be simultaneously solved for $\mathbf{u}(x, t, \lambda)$, then the function $\phi(x, t, \lambda)$ solves the Schrödinger equation with potential $V(x, t)$.

3. Require that the ODEs can be simultaneously solved for all $\lambda$. This places nonlinear constraints on the functions $q_{n}(x, t)$. In this case, the compatibility condition (5) yields the vector nonlinear Schrödinger equation for the components $q_{n}(x, t)$

$$
\mathrm{i} \partial_{t} q_{n}+\frac{1}{2} \partial_{x}^{2} q_{n}+\left(\sum_{j=1}^{N}\left|q_{j}\right|^{2}\right) q_{n}=0, \quad n=1, \ldots, N .
$$

This is the system studied by Manakov [2] in the case $N=2$. The nonlinear system (9) is integrable for all $N$ by virtue of its representation as the compatibility condition of the two linear problems of its Lax pair (3) with the matrices (8). Note that this Lax pair differs from the usual Lax pair for Eq. (9), posed for a vector $\mathbf{v}(x, t, \lambda)$, by an exponential gauge transformation: $\mathbf{v}(x, t, \lambda)=\mathbf{u}(x, t, \lambda) \exp \left(\mathrm{i}\left(\lambda x+\lambda^{2} t\right)\right)$. The reason we choose the gauge we do is precisely so that the first component of $\mathbf{u}$, the function $\phi(x, t, \lambda)$, satisfies (1). By contrast, the first component of the vector $\mathbf{v}$ does not satisfy any simple linear equations that do not contain $\lambda$.

We will call time-dependent potential functions of the form (7) where $\mathbf{q}(x, t)$ satisfies (9) separable. The three steps of the separation scheme above show that for separable potential functions it is again possible to identify a family of modes $\phi(x, t, \lambda)$ that satisfy the linear Schrödinger equation (1) for each $\lambda$, and whose spatial and temporal behaviors are independently specified by ODEs. Unlike those potentials satisfying (6), for which the more traditional method of separation of variables works, separable potential functions (7) can have complicated time dependence. The complexity available in the family of separable potentials increases with the number of components, $N$. In fact, there is some evidence [3] that a quite general (nonseparable) attractive potential $V(x, t)$ can be approximated pointwise in $x$ and $t$ by a sequence $\left\{V_{n}(x, t)\right\}$ of separable potentials. This pointwise convergence relies on allowing the number of components to increase.

Having a family of modes $\phi(x, t, \lambda)$ satisfying the time-dependent Schrödinger equation (1) is certainly useful, but it is not necessarily the case that these modes may be superposed to form the general solution for initial data 
$f_{0}(x)$ in some desired class. Of course, for well-behaved stationary potentials, $L_{2}$ completeness of states follows from the spectral theorem for self-adjoint operators [4]. Below, we intend to show that for a large sub-class of separable potentials, the scheme described above yields the solution of the initial value problem for (1) for absolutely continuous initial data $f_{0}(x)$ in $L_{1}$. This result will play the same role for these separable potentials that the spectral theorem plays for stationary potentials. It thus places a large set of time-dependent Schrödinger equations on the same footing as their stationary analogs.

The rest of this paper is organized as follows. In Section 2 the particular sub-class of separable potentials (7) corresponding to $M$-soliton solutions of the nonlinear system (9) will be introduced, and the corresponding modes $\phi(x, t, \lambda)$ will be constructed as well. Section 3 contains the main result of this paper, a proof that for fixed $t$, the modes $\phi(x, t, \lambda)$ may be uniquely superposed to expand any absolutely continuous $L_{1}$ function of $x$. In Section 4 we briefly show how to use this completeness relation to solve the initial value problem for (1), giving a modal expansion formula expressing the solution in terms of the initial data. We conclude in Section 5 by discussing some extensions to more general separable potentials of the form (7).

Some, but not all, of our results have been obtained by others using different methods. Nogami and Warke [5] have found several particular solutions to the linear equation (1) in their approach to multisoliton solutions of the nonlinear problem (9). Indeed, superpositions of the functions $q_{n}(x, t)$ themselves form an $N$-dimensional manifold of particular solutions of (1) when $V(x, t)$ is separable. Other authors [1,6] have exploited the well-known connection [7-10] between the time-dependent Schrödinger equation (1) and the Kadomtsev-Petviashvili (KP) equation to present families of particular solutions of (1) for certain potentials $V(x, t)$. The reason that both the vector nonlinear Schrödinger system (9) and the KP equation can be used to solve (1) is that the integrable dynamics of the former can be embedded within the latter for any number of components $N$ [3]. The works developing inversescattering machinery for the two-dimensional operator in (1) with a view toward linearizing the KP equation [7-10] suggest that there are enough eigenfunctions to expand many more functions of $x$ and $t$ than just the potential itself, and thus these techniques could likely reproduce or even extend our results. However, the advantage of using the vector nonlinear Schrödinger system (9) as the source of integrability of the potential is that its Lax pair representation allows the temporal evolution of each mode $\phi(x, t, \lambda)$ to be separated from its corresponding spatial description in a way that obviously generalizes the simple procedure that applies to stationary potentials.

\section{Algebraic construction of multisoliton separable potentials and modes}

In this section, we will present a sub-class of separable potentials $V(x, t)$ that can be specified along with their modes $\phi(x, t, \lambda)$ by algebraic operations only. These correspond to the $M$-soliton solutions of the nonlinear system (9). A basis of simultaneous solutions of the Lax pair is constructed in the following manner, a generalization for arbitrary $N$ of the familiar procedure for $N=1$ and $N=2$. Fix the number of components $N$, and choose an unrelated positive integer $M$. A fundamental solution matrix of (3) is sought in the form

$$
\mathbf{F}(x, t, \lambda)=\left(\lambda^{M} \mathbf{1}_{N+1}+\sum_{p=0}^{M-1} \lambda^{p} \mathbf{F}^{(p)}(x, t)\right)\left[\begin{array}{cc}
\exp \left(-2 \mathrm{i}\left(\lambda x+\lambda^{2} t\right)\right) & \mathbf{0}^{\mathrm{T}} \\
\mathbf{0} & \mathbf{1}_{N}
\end{array}\right] .
$$

Here, the symbol $\mathbf{1}_{D}$ denotes the $D \times D$ identity matrix. We denote the first column of $\mathbf{F}$ by $\mathbf{u}(x, t, \lambda)$ and the submatrix of remaining columns by $\mathbf{U}(x, t, \lambda)$. The matrix coefficients $\mathbf{F}^{(p)}(x, t)$ are determined by choosing $M$ distinct complex numbers $\lambda_{k}$ in the upper half plane, $M$ complex vectors $\mathbf{b}^{(k)}$ of $N$ components each, and then insisting that

$$
\mathbf{u}\left(x, t, \lambda_{k}\right)=\mathbf{U}\left(x, t, \lambda_{k}\right) \mathbf{b}^{(k)^{*}}, \quad \mathbf{U}\left(x, t, \lambda_{k}^{*}\right)=-\mathbf{u}\left(x, t, \lambda_{k}^{*}\right) \mathbf{b}^{(k) \mathrm{T}},
$$


for $k=1, \ldots, M$. Dimension counting arguments based on Liouville's theorem can then be employed to show that the columns of $\mathbf{F}$ will be simultaneous solutions of the Lax pair as long as one defines

$$
q_{n}(x, t)=2 \mathrm{i} F_{1, n+1}^{(M-1)}(x, t) .
$$

More precisely, the argument begins with the observation that the matrices $\left(\partial_{x}-\mathbf{L}\right) \mathbf{F}$ and $\left(\partial_{t}-\mathbf{B}\right) \mathbf{F}$ satisfy the relations (11) because $\mathbf{F}$ does. Liouville's theorem is used to show that the linear space of matrices having the form (10) up to a scalar multiple and satisfying relations (11) is one-dimensional. With the $q_{n}(x, t)$ chosen according to (12), one then finds that both $\left(\partial_{x}-\mathbf{L}\right) \mathbf{F}$ and $\left(\partial_{t}-\mathbf{B}\right) \mathbf{F}$ are in this one-dimensional linear space with leading coefficient zero (the latter part of the statement is shown by asymptotic analysis of (3) for large $\lambda$ ). This implies that the two matrices vanish identically, and that $\mathbf{F}$ is a simultaneous solution of the two linear problems. It then follows that the functions $q_{n}(x, t)$ satisfy the compatibility condition of (3), the vector nonlinear Schrödinger equation (9).

The first element of $\mathbf{u}(x, t, \lambda)$ is, for each $\lambda$, a solution of the linear Schrödinger equation (1) with potential function

$$
V(x, t)=-4 \sum_{n=1}^{N}\left|F_{1, n+1}^{(M-1)}(x, t)\right|^{2} .
$$

Explicitly, this element takes the form

$$
\phi(x, t, \lambda)=\left(\lambda^{M}+\sum_{p=0}^{M-1} \lambda^{p} F_{11}^{(p)}(x, t)\right) \exp \left(-2 \mathrm{i}\left(\lambda x+\lambda^{2} t\right)\right) .
$$

The data set $D_{N, M}=\left\{\lambda_{1}, \ldots, \lambda_{M}, \mathbf{b}^{(1)}, \ldots, \mathbf{b}^{(M)}\right\}$ thus yields, by solving the system of linear algebraic equations (11) for the elements of $\mathbf{F}^{(p)}(x, t)$, both an explicit separable potential $V(x, t)$ and a family of modes $\phi(x, t, \lambda)$ parametrized by $\lambda$.

A separable potential constructed in this manner generically models the interaction of $M$ moving potential wells. By straightforward asymptotic analysis of the defining relations (11) in a frame of reference moving with fixed velocity, one can show that as $t \rightarrow \pm \infty$, the potential takes the form

$$
V(x, t) \sim \sum_{k=1}^{M} V_{k}^{ \pm}(x, t),
$$

with

$$
V_{k}^{ \pm}(x, t)=-4 \rho_{k}^{2} \operatorname{sech}^{2}\left(2 \rho_{k}\left(x+2 \sigma_{k} t\right)-\delta_{k}^{ \pm}\right),
$$

where $\lambda_{k}=\sigma_{k}+\mathrm{i} \rho_{k}$ and where $\delta_{k}^{ \pm}$are constants that depend on the elements of the vectors $\mathbf{b}^{(j)}$. This asymptotic expression holds as long as the velocities $\sigma_{k}$ are distinct; some results for nondistinct velocities appear in [11,12]. Each of the asymptotic component wells $V_{k}^{ \pm}(x, t)$ has a single bound state. The way that these bound states interact as the wells come together for finite $t$ can be investigated [13,14], yielding the result that a superposition of the isolated bound states prior to interaction is always converted into a superposition of isolated bound states after the interaction, with no loss. Explicit asymptotic calculations carried out for large $|t|$ give expressions for the field amplitude in each well as $t \rightarrow+\infty$ in terms of the corresponding amplitudes as $t \rightarrow-\infty$. Waves that are not confined to the potential wells as $t \rightarrow-\infty$ are scattered and lost.

On the other hand, this asymptotic analysis says nothing about the behavior of waves moving in the potential $V(x, t)$ for finite $x$ and $t$, especially in regions where the $M$ solitons in $\mathbf{q}(x, t)$ are interacting nonlinearly. During the interaction, it is not clear what kind of waves are bound states that will ultimately be captured by the wells $V_{k}^{+}(x, t)$, 
and what kind of waves will ultimately be scattered. The problem of understanding wave propagation for finite $x$ and $t$ is of special interest because we expect separable $M$-soliton potentials to uniformly approximate arbitrary attractive potentials in compact subsets of the $(x, t)$ plane. Solving the initial value problem for the Schrödinger equation in these regions will be possible after we have established the completeness relation for the modes $\phi(x, t, \lambda)$ in the next section.

\section{Completeness of states}

In this section, we will show that, for fixed $t$, there are enough modes $\phi(x, t, \lambda)$ to expand any absolutely continuous function $f(x)$ in $L_{1}$. We will also give explicit formulas for the unique expansion coefficients. This completeness relation is the tool that we will need to be able to express an arbitrary initial condition as a superposition of modes and hence solve the initial value problem for the linear Schrödinger equation (1).

Define the weight function $w(\lambda)$ by

$$
w(\lambda) \doteq \frac{1}{\pi} \prod_{k=1}^{M}\left(\lambda-\lambda_{k}\right)^{-1}\left(\lambda-\lambda_{k}^{*}\right)^{-1} .
$$

Also, denote the residues by

$$
w_{k} \doteq \lim _{\lambda \rightarrow \lambda_{k}^{*}}\left(\lambda-\lambda_{k}^{*}\right) w(\lambda)
$$

Theorem 1. Let $t$ be fixed but arbitrary, and let $f(x) \in L_{1}$ be absolutely continuous. For real $\lambda$, set

$$
\hat{f}(\lambda) \doteq \int_{-\infty}^{\infty} f(x) \phi(x, t, \lambda)^{*} \mathrm{~d} x
$$

and for $k=1, \ldots, M$, set

$$
\hat{f_{k}} \doteq \int_{-\infty}^{\infty} f(x) \phi\left(x, t, \lambda_{k}\right)^{*} \mathrm{~d} x
$$

Then

$$
f(x)=\lim _{R \rightarrow \infty} \int_{-R}^{R} \hat{f}(\lambda) w(\lambda) \phi(x, t, \lambda) \mathrm{d} \lambda+2 \pi \mathrm{i} \sum_{k=1}^{M} \hat{f}_{k} w_{k} \phi\left(x, t, \lambda_{k}^{*}\right) .
$$

Remark. The fact that the modes $\phi(x, t, \lambda)$ form a complete set in this sense is suggested by the fact that for large $\lambda, \phi(x, t, \lambda)$ behaves as a family of exponential functions:

$$
\phi(x, t, \lambda)=\lambda^{M} \exp \left(-2 \mathrm{i}\left(\lambda x+\lambda^{2} t\right)\right)\left(1+\mathrm{O}\left(|\lambda|^{-1}\right)\right),
$$

which are known to be complete. Roughly speaking, the proof is based on this observation. Similar arguments have been used successfully to prove completeness of other families of functions [15]. 
Proof. For fixed $x$ and $t, \phi(x, t, \lambda)$ is an entire function of $\lambda$, with behavior for large $\lambda$ given by (22). Thus, for finite $z$, both integrals

$$
I_{<}(t, z, \lambda)=\int_{-\infty}^{z} f(x) \phi\left(x, t, \lambda^{*}\right)^{*} \mathrm{~d} x, \quad I_{>}(t, z, \lambda)=\int_{z}^{\infty} f(x) \phi\left(x, t,-\lambda^{*}\right)^{*} \mathrm{~d} x,
$$

are analytic for $\operatorname{Im}(\lambda)<0$. Now, for $R>\max \left|\lambda_{k}\right|$ set

$$
g_{R}(x, t, z)=\int_{C_{R}^{-}}\left[w(\lambda) \phi(x, t, \lambda) I_{<}(t, z, \lambda)+w(-\lambda) \phi(x, t,-\lambda) I_{>}(t, z, \lambda)\right] \mathrm{d} \lambda,
$$

where $C_{R}^{-}$is the contour from $-R$ to $R$ along the circle of radius $R$ in the lower half plane.

Lemma 1. The function $g_{R}(x, t, z)$ is independent of $z$.

Proof of Lemma 1. Using Fubini's theorem to exchange the order of integration, we have

$$
g_{R}\left(x, t, z_{2}\right)-g_{R}\left(x, t, z_{1}\right)=\int_{z_{1}}^{z_{2}} f(y) I(x, y, t) \mathrm{d} y,
$$

where

$$
I(x, y, t)=\int_{C_{R}} \phi(x, t, \lambda) \phi\left(y, t, \lambda^{*}\right)^{*} w(\lambda) \mathrm{d} \lambda,
$$

and $C_{R}$ is the positively oriented circular closed contour of radius $R$ centered at $\lambda=0$. Let us show that $I(x, y, t)$ vanishes by evaluating the integral using the residue theorem. Because $\phi(x, t, \lambda)$ is entire, all poles of the integrand are contributed by the weight function $w(\lambda)$. Explicitly

$$
\begin{aligned}
I(x, y, t)= & 2 \pi \mathrm{i} \sum_{k=1}^{M}\left(\lambda_{k}^{M}+\sum_{p=0}^{M-1} \lambda_{k}^{p} F_{11}^{(p)}(x, t)\right)\left(\lambda_{k}^{M}+\sum_{p=0}^{M-1} \lambda_{k}^{p} F_{11}^{(p)}(y, t)^{*}\right) w_{k}^{*} \exp \left(-2 \mathrm{i} \lambda_{k}(x-y)\right) \\
& +\left(\lambda_{k}^{* M}+\sum_{p=0}^{M-1} \lambda_{k}^{* p} F_{11}^{(p)}(x, t)\right)\left(\lambda_{k}^{* M}+\sum_{p=0}^{M-1} \lambda_{k}^{* p} F_{11}^{(p)}(y, t)^{*}\right) w_{k} \exp \left(-2 \mathrm{i} \lambda_{k}^{*}(x-y)\right) .
\end{aligned}
$$

Eliminating $F_{1,2}^{(p)}(x, t), \ldots, F_{1, N+1}^{(p)}(x, t)$ from relations (11) gives

$$
\begin{aligned}
& \left(\lambda_{j}^{M}+\sum_{p=0}^{M-1} \lambda_{j}^{p} F_{11}^{(p)}(x, t)\right) \exp \left(-2 \mathrm{i}\left(\lambda_{j} x+\lambda_{j}^{2} t\right)\right) \\
& =-\sum_{m=1}^{M} \sum_{k=1}^{M} \lambda_{j}^{m-1} h_{m k}^{*} \beta_{k j}\left(\lambda_{k}^{* M}+\sum_{p=0}^{M-1} \lambda_{k}^{* p} F_{11}^{(p)}(x, t)\right) \exp \left(-2 \mathrm{i}\left(\lambda_{k}^{*} x+\lambda_{k}^{* 2} t\right)\right),
\end{aligned}
$$

where $h_{m k}$ are the elements of the inverse of the Vandermonde matrix

$$
\Lambda=\left[\begin{array}{cccc}
1 & \lambda_{1} & \cdots & \lambda_{1}^{M-1} \\
\vdots & \vdots & & \vdots \\
1 & \lambda_{M} & \cdots & \lambda_{M}^{M-1}
\end{array}\right]
$$


and $\beta_{k j}=\mathbf{b}^{(k) \mathrm{T}} \mathbf{b}^{(j)^{*}}$ are the elements of a Hermitean matrix. Then, we can write

$$
I(x, y, t)=2 \pi \mathrm{i} \sum_{k=1}^{M} \sum_{m=1}^{M}\left\{\alpha_{k m}+\alpha_{m k}^{*}\right\} \gamma_{m k}(x, y, t),
$$

where

$$
\alpha_{k m}=-w_{k}^{*} \sum_{j=1}^{M} \lambda_{k}^{j-1} h_{j m}^{*}
$$

and

$$
\begin{aligned}
\gamma_{m k}(x, y, t)= & \beta_{m k}\left(\lambda_{m}^{* M}+\sum_{p=0}^{M-1} \lambda_{m}^{* p} F_{11}^{(p)}(x, t)\right)\left(\lambda_{k}^{M}+\sum_{p=0}^{M-1} \lambda_{k}^{p} F_{11}^{(p)}(y, t)^{*}\right) \\
& \times \exp \left(-2 \mathrm{i}\left(\lambda_{m}^{*} x+\lambda_{m}^{* 2} t\right)\right) \exp \left(2 \mathrm{i}\left(\lambda_{k} x+\lambda_{k}^{2} t\right)\right) .
\end{aligned}
$$

Thus, it suffices to show that $\alpha_{k m}+\alpha_{m k}^{*}=0$, a condition that depends only on the numbers $\lambda_{k}$. This condition is equivalent to the statement that the matrix

$$
\Lambda^{\mathrm{T}} \operatorname{diag}\left(w_{1}^{*}, \ldots, w_{M}^{*}\right) \Lambda
$$

is skew-Hermitean. But this matrix is obviously symmetric, and it can be shown that its elements are all imaginary. This ends the proof of Lemma 1.

Now define $f_{R}(x)$ by

$$
f_{R}(x)=\frac{1}{\pi} \int_{C_{R}^{-}}\left[\int_{-\infty}^{x} f(y) \exp (-2 \mathrm{i} \lambda(x-y)) \mathrm{d} y+\int_{x}^{\infty} f(y) \exp (2 \mathrm{i} \lambda(x-y)) \mathrm{d} y\right] \mathrm{d} \lambda .
$$

It follows from the Fourier inversion theorem that

$$
\lim _{R \rightarrow \infty}\left|f(x)-f_{R}(x)\right|=0 .
$$

Lemma 2. Let $x, t$, and $z$ be fixed. Then

$$
\lim _{R \rightarrow \infty}\left|g_{R}(x, t, z)-f_{R}(x)\right|=0 .
$$

Proof of Lemma 2. By Lemma 1, it suffices to consider $z=x$. We have

$$
\begin{aligned}
g_{R}(x, t, x)-f_{R}(x)= & \int_{C_{R}^{-}}\left[\int_{-\infty}^{x} Q(x, y, t, \lambda) f(y) \exp (-2 \mathrm{i} \lambda(x-y)) \mathrm{d} y\right. \\
& \left.+\int_{x}^{\infty} Q(x, y, t,-\lambda) f(y) \exp (2 \mathrm{i} \lambda(x-y)) \mathrm{d} y\right] \mathrm{d} \lambda,
\end{aligned}
$$

where

$$
Q(x, y, t, \lambda) \doteq \phi(x, t, \lambda) \phi\left(y, t, \lambda^{*}\right)^{*} w(\lambda) \exp (2 \mathrm{i} \lambda(x-y))-\frac{1}{\pi} .
$$


It follows from relations (11) that both $Q(x, y, t, \lambda)$ and $\partial_{y} Q(x, y, t, \lambda)$ are $\mathrm{O}\left(|\lambda|^{-1}\right)$ uniformly in $y$ for large $\lambda$. Integrating by parts,

$$
\begin{aligned}
g_{R}(x, t, x)-f_{R}(x)= & \int_{C_{R}^{-}}[\{(x, x, t, \lambda)+Q(x, x, t,-\lambda)\} f(x) \\
& -\int_{-\infty}^{x}\left\{\partial_{y} Q(x, y, t, \lambda) \cdot f(y)+Q(x, y, t, \lambda) f^{\prime}(y)\right\} \exp (-2 \mathrm{i} \lambda(x-y)) \mathrm{d} y \\
& \left.+\int_{x}^{\infty}\left\{\partial_{y} Q(x, y, t,-\lambda) \cdot f(y)+Q(x, y, t,-\lambda) f^{\prime}(y)\right\} \exp (2 \mathrm{i} \lambda(x-y)) \mathrm{d} y\right] \frac{\mathrm{d} \lambda}{2 \mathrm{i} \lambda} .
\end{aligned}
$$

From our choice of $z=x$, the exponentials are all bounded by 1 , and $|\lambda|=R$ on the contour $C_{R}^{-}$of length $\pi R$, so since $f(x)$ is an absolutely continuous $L_{1}$ function,

$$
\begin{aligned}
\left|g_{R}(x, t, x)-f_{R}(x)\right| \leq & \frac{\pi}{2}\left[|f(x)| \sup _{\lambda \in C_{R}^{-}}\{|Q(x, x, t, \lambda)|+|Q(x, x, t,-\lambda)|\}\right. \\
& +\|f\|_{1} \sup _{\lambda \in C_{R}^{-}} \sup _{y}\left\{\left|\partial_{y} Q(x, y, t, \lambda)\right|+\left|\partial_{y} Q(x, y, t,-\lambda)\right|\right\} \\
& \left.+\left\|f^{\prime}\right\|_{1} \sup _{\lambda \in C_{R}^{-}} \sup _{y}\{|Q(x, y, t, \lambda)|+|Q(x, y, t,-\lambda)|\}\right],
\end{aligned}
$$

which vanishes for large $R$ by our uniform estimates on $Q$. This proves Lemma 2 .

Because $f_{R}(x)$ converges to $f(x)$, we now have the result that for any fixed $x, z$, and $t$,

$$
f(x)=\lim _{R \rightarrow \infty} g_{R}(x, t, z) .
$$

Applying the residue theorem to $g_{R}(x, t, z)$ to bring the contour $C_{R}^{-}$to the real axis gives discrete contributions from $\lambda=\lambda_{k}^{*}$ and $\lambda=-\lambda_{k}$ :

$$
\begin{aligned}
g_{R}(x, t, z)= & \int_{-R}^{R} w(\lambda) \phi(x, t, \lambda) I_{<}(t, z, \lambda) \mathrm{d} \lambda+\int_{-R}^{R} w(-\lambda) \phi(x, t,-\lambda) I_{>}(t, z, \lambda) \mathrm{d} \lambda \\
& +2 \pi \mathrm{i} \sum_{k=1}^{M}\left[\phi\left(x, t, \lambda_{k}^{*}\right) I_{<}\left(t, z, \lambda_{k}^{*}\right) w_{k}-\phi\left(x, t, \lambda_{k}\right) I_{>}\left(t, z,-\lambda_{k}\right) w_{k}^{*}\right] .
\end{aligned}
$$

Using the symmetry of the integrand on the real axis the first terms can be combined:

$$
\begin{aligned}
g_{R}(x, t, z)= & \int_{-R}^{R} \hat{f}(\lambda) w(\lambda) \phi(x, t, \lambda) \mathrm{d} \lambda \\
& +2 \pi \mathrm{i} \sum_{k=1}^{M}\left[\phi\left(x, t, \lambda_{k}^{*}\right) I_{<}\left(t, z, \lambda_{k}^{*}\right) w_{k}-\phi\left(x, t, \lambda_{k}\right) I_{>}\left(t, z,-\lambda_{k}\right) w_{k}^{*}\right] .
\end{aligned}
$$


Finally, we know from Lemma 1 that $g_{R}(x, t, z)$ is independent of $z$, and since we no longer require any analytic properties of the integrand, we can pass to the limit $z \rightarrow+\infty$. This yields the desired expression

$$
g_{R}(x, t,+\infty)=\int_{-R}^{R} \hat{f}(\lambda) w(\lambda) \phi(x, t, \lambda) \mathrm{d} \lambda+2 \pi \mathrm{i} \sum_{k=1}^{M} \hat{f}_{k} w_{k} \phi\left(x, t, \lambda_{k}^{*}\right),
$$

and completes the proof of Theorem 1.

\section{Solution of the initial value problem}

Let us now describe how to use the completeness relation established above to solve the initial value problem for the Schrödinger equation (1) for separable $M$-soliton potentials. Suppose that $f(x, t)$ satisfies the linear Schrödinger equation (1) with potential (7), and that $f(x, t)$ and $\partial_{x} f(x, t)$ both vanish as $x \rightarrow \pm \infty$. Then, using (1) and integrating by parts, it is easy to show that the transform quantities

$$
\hat{f}(\lambda) \doteq \int_{-\infty}^{\infty} f(x, t) \phi(x, t, \lambda)^{*} \mathrm{~d} x \quad \hat{f}_{k} \doteq \int_{-\infty}^{\infty} f(x, t) \phi\left(x, t, \lambda_{k}\right)^{*} \mathrm{~d} x, \quad k=1, \ldots, M,
$$

are independent of $t$. They may thus be calculated from the initial data $f(x, 0)=f_{0}(x)$. As long as the solution for this initial data is unique and remains in the class of absolutely continuous $L_{1}$ functions of $x$, it is given by the modal expansion formula

$$
f(x, t)=\lim _{R \rightarrow \infty} \int_{-R}^{R} \hat{f}(\lambda) w(\lambda) \phi(x, t, \lambda) \mathrm{d} \lambda+2 \pi \mathrm{i} \sum_{k=1}^{M} \hat{f}_{k} w_{k} \phi\left(x, t, \lambda_{k}^{*}\right) .
$$

This formula explicitly expresses the solution $f(x, t)$ of the initial value problem for (1) in terms of the initial data $f_{0}(x)$. The discrete part of the formula represents solution components that for large $|t|$ are confined to the individual moving potential wells $V_{k}^{ \pm}(x, t)$, while the integral term describes waves that scatter from the potential $V(x, t)$. Although mathematically distinct for all $t$, the two parts of the formula are difficult to distinguish from each other in regions where the solitons in $\mathbf{q}(x, t)$ are not well-separated.

\section{Conclusion}

The arguments above show that the initial value problem for the linear Schrödinger equation (1) with a separable $M$-soliton potential $V(x, t)$ - which can have very complicated time dependence, especially in the interaction region of the solitons - can be solved by separation of variables just as in the case of stationary potentials $V=V(x)$. In a sense, the only new feature is that in the present case, the mode $\phi(x, t, \lambda)$ cannot be expressed as a simple product of a function of $x$ and a function of $t$.

The $M$-soliton potentials considered in detail in this paper are, however, a subset of all separable potentials, and in some sense our results should extend to separable potentials that come from rapidly decaying solutions $\mathbf{q}(x, t)$ of (9) for which there is a nonzero reflection coefficient in the inverse-scattering transform domain. It is a familiar but imprecise notion that the $M$-soliton solutions of the vector nonlinear Schrödinger equation (9) are dense in the set of all rapidly decaying solutions, with radiative components of the solution encoded in a nonzero reflection 
coefficient being somehow represented as a "sea of solitons". Unfortunately, the presence of a nonzero reflection coefficient introduces genuine technical difficulties in extending our completeness proof to more general separable potentials. Because no longer there exists a single mode function $\phi(x, t, \lambda)$ that is analytic in the whole $\lambda$ plane, one introduces the Jost solutions of the linear problems (3) making up the Lax pair. In particular, one finds functions satisfying $(1), \phi^{+}(x, t, \lambda)$, analytic for $\operatorname{Im}(\lambda)<0$ and $\phi^{-}(x, t, \lambda)$, analytic for $\operatorname{Im}(\lambda)>0$. These functions are further characterized as being eigenfunctions, with eigenvalue $\lambda$, of the operators $L^{ \pm}$with

$$
\left(L^{+} f\right)(x)=-\frac{1}{2 \mathrm{i}} \partial_{x} f(x)+\frac{1}{2 \mathrm{i}} \mathbf{q}(x, t)^{\mathrm{T}} \int_{x}^{\infty} \mathbf{q}(y, t)^{*} f(y) \mathrm{d} y,
$$

and with $L^{-}$being the $L_{2}$ adjoint of $L^{+}$. One constructs from these two modal functions the quantity that is the analog of $g_{R}(x, t, z)$, and must prove that it (or perhaps its limit for large $R$ ) is independent of $z$. This step is crucial in controlling the exponential growth for large $\lambda$, since it allows us to consider at first $z=x$, where there is no growth at all, and then after the fact to send $z$ to $+\infty$. The proof of $z$ independence must follow a different path, since the presence of a reflection coefficient means that it will no longer suffice to calculate residues. We expect to overcome this technical difficulty and present the completeness relation for the full set of rapidly decreasing separable potentials in a future publication.

A related direction of research is the precise development of the approximation theory that will allow Schrödinger equations with arbitrary attractive and decaying time-dependent potentials $V(x, t)$ to be identified with sequences of separable equations. Such a theory would be a powerful tool in the analysis of solutions to general Schrödinger equations in compact regions of the $(x, t)$ plane.

Another path we would like to follow is the consideration of alternative splittings of the Schrödinger equation (1) that fall into the same framework as described in Section 1. One such splitting is closely related to that described in this paper, and yields the defocusing variant of the vector nonlinear Schrödinger equation (9) as the compatibility condition. There is a class of algebraic potentials $V(x, t)$ in this case as well, corresponding to the interactions of $M$ dark solitons in the vector-valued field $\mathbf{q}(x, t)$. After subtracting a constant background potential, these potentials also represent interacting potential wells. The modes $\phi(x, t, \lambda)$ can be found explicitly, and have been studied [16]. Proving completeness of states in this case will require deforming contours on Riemann surfaces that are $(N+1)$-sheeted genus 0 coverings of the $\lambda$-plane. The resulting completeness relation involves integrals on several sheets of the surface and will be given elsewhere. We also suspect that other useful splittings may be possible, with compatibility conditions that do not resemble the vector nonlinear Schrödinger equation at all.

\section{References}

[1] B.A. Dubrovin, T.M. Malanyuk, I.M. Krichever, V.G. Makhan'kov, Exact solutions of the time-dependent Schrödinger equation with self-consistent potentials, Sov. J. Part. Nucl. 19 (1988) 252-269.

[2] S.V. Manakov, On the theory of two-dimensional stationary self-focusing of electromagnetic waves, Sov. Phys. JETP 38 (1974) $248-253$.

[3] P.D. Miller, Vector nonlinear Schrödinger hierarchies as approximate Kadomtsev-Petviashvili hierarchies, Physica D 101 (1997) $17-26$.

[4] F. Riesz, B.Sz. Nagy, Functional Analysis, Dover, New York, 1990.

[5] Y. Nogami, C.S. Warke, Soliton solutions of multicomponent nonlinear Schrödinger equation, Phys. Lett. A 59 (1976) $251-253$.

[6] M.J. Ablowitz, J. Villarroel, Solutions to the time dependent Schrödinger and the Kadomtsev-Petviashvili equations, Phys. Rev. Lett. 78 (1997) 570-573.

[7] S.V. Manakov, The inverse scattering transform for the time-dependent Schrödinger equation and Kadomtsev-Petviashvili equation, Physica D 3 (1) (1981) 420-427.

[8] A.S. Fokas, M.J. Ablowitz, On the inverse scattering and direct linearizing transforms for the Kadomtsev-Petviashvili equation, Phys. Lett. A 94 (1983) 67-70. 
[9] A.S. Fokas, M.J. Ablowitz, On the inverse scattering of the time-dependent Schrödinger equation and the associated KadomtsevPetviashvili (I) equation, Stud. Appl. Math. 69 (1983) 211-228.

[10] X. Zhou, Inverse scattering transform for the time dependent Schrödinger equation with applications to the KPI equation, Comm. Math. Phys. 128 (1990) 551-564.

[11] J.A. Besley, N.N. Akhmediev, P.D. Miller, Modes of periodic waveguides, Opt. Lett. 22 (1997) 1162-1164.

[12] J.A. Besley, N.N. Akhmediev, P.D. Miller, Periodic optical waveguides: exact Floquet theory and spectral properties, Stud. Appl. Math., to appear.

[13] P.D. Miller, N.N. Akhmediev, Transfer matrices for multiport devices made from solitons, Phys. Rev. E 53 (1996) $4098-4106$.

[14] P.D. Miller, N.N. Akhmediev, Do solitons exchange conserved quantities during collisions? Phys. Rev. Lett. 76 (1996) 38-41.

[15] R.L. Sachs, Completeness of derivatives of squared Schrödinger eigenfunctions and explicit solutions of the linearized KdV equation, SIAM J. Math. Anal. 14 (1983) 674-683.

[16] P.D. Miller, Zero-crosstalk junctions made from dark solitons, Phys. Rev. E 53 (1996) 4137-4142. 